\title{
Micro-scale characterization of fluid flow in a uniform particle pack using coupled discrete element-lattice Boltzmann method
}

\author{
M. Aminpour \& S. A. Galindo-Torres \& A. Scheuermann \& L. Li \\ Geotechnical Engineering Centre. School of Civil Engineering, The University of Queensland, \\ Brisbane QLD 4072, Australia
}

\begin{abstract}
Particle scale investigation of geo-mechanical phenomena using numerical simulations has increasingly attracted the attention of researchers stimulated by recent advances in computational capacities. In this paper, the pore scale characteristics of flow in a particulate soil pack is investigated. A randomly distributed pack of uniform particles is created using a discrete element simulation of 500 particles with $9 \mathrm{~mm}$ diameter settling freely into a box. Then the lattice-Boltzmann method is utilized to simulate a constant flow passing through the particle pack with a wide range of Reynolds numbers from $10^{-4}$ to 18 . Subsequently, the pressure loss occurred in the flow when it passes through the particles is studied to characterize the transformation of flow conditions from laminar to turbulent mode. The pore scale characteristics of the flow, e.g. fow tortuosity, are then compared to those of pack scale, $e . g$. fluid pressure difference between base and top of the particle pack to acquire more understanding on transforming from laminar to turbulent flow in micro (pore scale) and macro (pack) levels. Furthermore, the effects of flow rates demonstrated by Reynolds numbers are examined on the drag forces applied on particles. Discussions on the micro-scale behaviour of flow in porous media based on the results obtained from numerical simulations are provided.
\end{abstract}

\section{INTRODUCTION}

Fluid dynamics in porous media have been continuously investigated by researchers considering its importance in various fields of science and technology, such as pharmacological engineering, petroleum engineering, ground water hydrology, agricultural engineering and soil mechanics. New advancements in computational capacities have provided researchers with new levels of possibilities to examine and understand the physical phenomena in details. Observing the microscopic behaviour of flow in complex structures like particulate porous media is one example for what computational advances could present which is still much more difficult to be investigated through experiments.

Known as a governing parameter to describe the complexity of micro-structures, tortuosity is a key factor to characterize the transport properties of porous media. While the macroscopic transport properties are characterised by parameters like permeability, electric conductivity and diffusion conductivity, they are directly affected by the tortuosity of the flow (Saomoto and Katagiri 2015).

Variation of the flow tortuosity has been frequently investigated, either analytically (e.g. Guo 2015) or numerically (e.g. Duda et al. 2011, Saomoto and
Katagiri 2015), particularly as a function of porosity (e.g. Matyka et al. 2008, Xiao-Wu et al. 2012, Sun et al. 2013, Freytag and Roque 2013, Wang 2014). To the knowledge of authors, the previous investigations, however, have neglected the potential effects of velocity ranges on the tortuosity calculations. The present study is aimed to address the micro-scale characterisation of flow in a typical porous medium examined in a wide range of flow velocities, represented as Reynolds numbers, with an especial focus on the flow tortuosity. An extensive range of flow conditions from pre- to the post-Darsy is covered. In addition, the study will discuss the variation of drag forces imposed to the particles, varying with the flow velocity, which is directly attributed to the erosion phenomenon in particulate porous media.

\section{METHODOLOGY}

The following sections will briefly describe the discrete element method (DEM), and the Lattice Boltzmann method (LBM) which are used in a coupled implementation approach in this study. The original method is fully described by Galindo-Torres 2013. 
The Mechsys open source library ${ }^{1}$ includes the associated algorithm.

\subsection{Discrete Element Method (DEM)}

The standard linear dashpot model introduced by Cundall and Strack 1979 is the basic formulation of the DEM used in this study. Discrete elements are freely moving inside the domain while a contact would be defined once two particles intersect. The overlapping caused by this intersection is characterised by overlapping length $\left(\delta_{n}\right)$, by which a normal force $\overrightarrow{\boldsymbol{F}}_{n}$ between the particles can be defined in the normal direction $\overrightarrow{\hat{n}}$,

$\overrightarrow{\boldsymbol{F}}_{n}=K_{n} \delta_{n} \overrightarrow{\hat{\boldsymbol{n}}}$,

where $K_{n}$ or normal stiffness is a parameter determined using the elastic modulus of the material.

The middle point of the overlapping length is defined as the point of contact where both contacting particles could rotate around, causing a relative velocity, $\overrightarrow{\boldsymbol{v}}_{r e l}$, representing the relative displacement of the particles at the contact point. The tangential component of the relative velocity, $\overrightarrow{\boldsymbol{v}}_{t}$, is then integrated to provide the tangential displacement, $\delta_{t}$. Using this displacement component, the tangential force, $\overrightarrow{\boldsymbol{F}}_{t}$, along the tangential plane to the overlap of particles is calculated as follows:

$$
\overrightarrow{\boldsymbol{F}}_{t}=K_{t} \delta_{t} \overrightarrow{\hat{\boldsymbol{t}}}
$$

where $\overrightarrow{\hat{\boldsymbol{t}}}=\overrightarrow{\boldsymbol{v}}_{t} / v_{t}$ and $K_{t}$ is a tangential stiffness. The provided tangential force represent the impact of particle surface roughness. The tangential force, however must not exceed the Coulomb limit (Galindo-Torres et al. 2012), thus chosen as the $\min \left(K_{t} \delta_{t}, \mu F_{n}\right) \overrightarrow{\hat{\boldsymbol{t}}}$ which depends to the microscopic friction coefficient $\mu$.

The energy is dissipated due to inclusion of the viscous forces,

$\overrightarrow{\boldsymbol{F}}_{v}=G_{n} m_{e} v_{n} \overrightarrow{\hat{\boldsymbol{n}}}+G_{t} m_{e} v_{t} \overrightarrow{\hat{\boldsymbol{t}}}$

where $G_{n}$ and $G_{t}$ are two dissipation constants for the normal and tangential directions and $m_{e}$ is the effective mass of the particle pair.

The resulting forces and the produced torques are then added to the forces produced by fluid-solid interaction (see the following section) updated step by step, in the numerical iterations. The equations of linear motion (Newton's second law) and angular motion (Euler's equations) are numerically integrated by the leap frog method (Wang et al. 2006), to simulate the fluid flow in the particulate medium.

\footnotetext{
${ }^{1}$ http://mechsys.nongnu.org/index.shtml
}

\subsection{Lattice-Boltzmann Method (LBM)}

A scheme called D3Q15 for the Lattice Boltzmann method is employed in this study (Hecht and Harting 2010). A cubic domain is defined covering the whole space where cells are representing the fluid particles. Each cell is specified with a set of probability distribution functions, $f_{i}$ representing the density of fluid particles going through the 15 discrete directions with the velocities of $\overrightarrow{\boldsymbol{e}}_{i}$ (see figure. 1). The density $\rho$ and velocity $\overrightarrow{\boldsymbol{u}}$ at each cell position $\overrightarrow{\boldsymbol{x}}$ is then determined as follows:

$$
\begin{array}{ll}
\rho(\overrightarrow{\boldsymbol{x}}) & =\sum_{i=0}^{15} f_{i}(\overrightarrow{\boldsymbol{x}}) \\
\overrightarrow{\boldsymbol{u}}(\overrightarrow{\boldsymbol{x}}) & =\frac{\sum_{i=0}^{15} f_{i}(\overrightarrow{\boldsymbol{x}}) \overrightarrow{\boldsymbol{e}}_{i}}{\rho(\overrightarrow{\boldsymbol{x}})}
\end{array}
$$

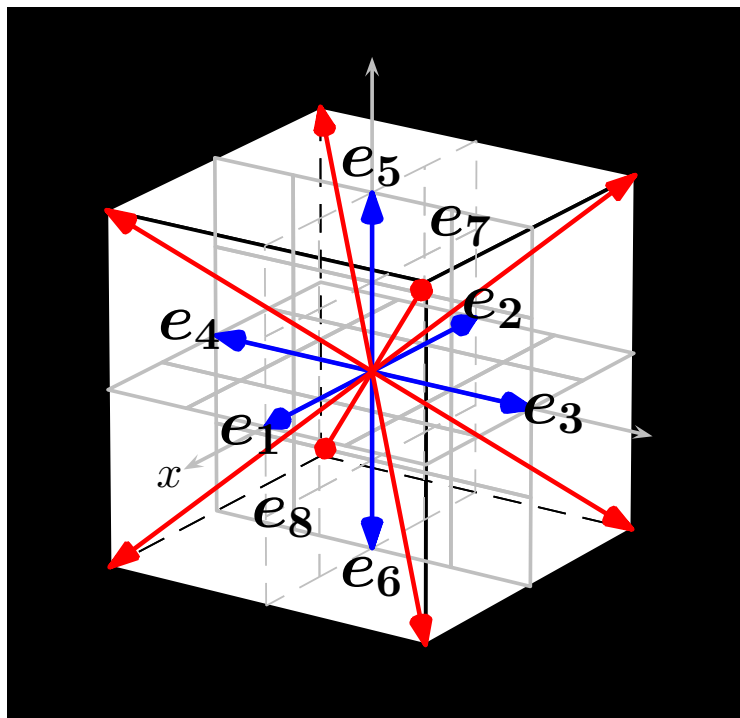

Figure 1: The fluid cell demonstrated according to D3Q15 LBM scheme showing the 15 discrete velocity directions

The distribution functions are evolved with the following equation derived from the Chapman Enskog expansion of the Boltzmann equation (Thorne \& Michael 2006),

$f_{i}\left(\overrightarrow{\boldsymbol{x}}+\overrightarrow{\boldsymbol{e}}_{i} \delta_{t}, t+\delta_{t}\right)=f_{i}(\overrightarrow{\boldsymbol{x}}, t)+\Omega_{c o l}$,

where $\overrightarrow{\boldsymbol{x}}$ is the position of the cell, $\delta_{t}$ is the time step and $\Omega_{c o l}$ is a collision operator representing the relaxation processes due to the collision of the fluid particles.

The widely used Bhatnagar-Gross-Krook (BGK) model (Qian, d'Humières, \& Lallemand 1992) for the collision operator is employed in the present study. It assumes the collision process bringing the system into an equilibrium state described by the following equilibrium function:

$\Omega_{c o l}=\frac{\delta t}{\tau}\left(f_{i}^{e q}-f_{i}\right)$

where $\tau$ is the characteristic relaxation time. The fluid flow Navier Stokes (NS) equations would be recovered (He and Luo 1997), if,

$$
f_{i}^{e q}=\omega_{i} \rho\left(1+3 \frac{\overrightarrow{\boldsymbol{e}}_{i} \cdot \overrightarrow{\boldsymbol{u}}}{C^{2}}+\frac{9\left(\overrightarrow{\boldsymbol{e}}_{i} \cdot \overrightarrow{\boldsymbol{u}}\right)^{2}}{2 C^{4}}-\frac{3 u^{2}}{2 C^{2}}\right)
$$


where the kinetic viscosity of the fluid $\nu$ is defined by,

$\nu=(\tau-0.5) \frac{\delta_{x}^{2}}{3 \delta_{t}}$

with $C=\delta_{x} / \delta_{t}$ being a characteristic lattice velocity given by the grid spacing $\delta_{x}$. The choice of $\tau$ is then constrained by the Eq. 8 as to be greater than 0.5 for the viscosity to be physically correct. Moreover, the values close to 0.5 would lead to numerically unstable behaviour due to non-linearity of NS equations (Thorne \& Michael 2006); therefore choosing $\tau$ values close to 1.0 is recommended.

LBM is formulated to model compressible fluids, whereas the equation of state would relate the density and the pressure $p$ at a given cell. The ideal gas law is such a equation which is used for this study:

$p=\frac{C^{2}}{3} \rho$,

where $C / \sqrt{3}$ is defined as the speed of sound of the simulated fluid. A perfect incompressibility is not achievable in this LBM scheme, while the fluid is assumed to be incompressible, when the density variation is negligible compared to the average density.

A net force is introduced for each cell to represent the body forces, e.g. the gravity force (Martys and Chen 1996). The velocity of flow is then modified with the following equation in the presence of body forces, $\overrightarrow{\boldsymbol{F}}_{b}$ :

$\overrightarrow{\boldsymbol{u}}^{\prime}=\overrightarrow{\boldsymbol{u}}+\frac{\delta_{t} \overrightarrow{\boldsymbol{F}}_{b}}{\rho}$

For the gravitational forces, the body force simply equals to $\overrightarrow{\boldsymbol{F}}_{g}=\rho \overrightarrow{\boldsymbol{g}}$ where $\overrightarrow{\boldsymbol{g}}$ is the gravitational acceleration (Buick and Greated 2000).

The constant velocity boundary conditions at inlet and outlet of the model to simulate the constant flow discharge is defined according to the formulation explained in the related LBM literature (for example, Zou and He 1997).

\section{MODEL SETUP}

The discrete element method was utilized to generate the particulate porous medium. The model consists of 500 particles, each $9 \mathrm{~mm}$ in diameter, randomly distributed into a box with a cross section of $10 \times 10 \mathrm{~cm}$. In order to obtain a naturally compacted arrangement of the particles, they are initially distributed randomly throughout the space above the box, then allowed to deposit into the box through a free fall simulation (see figure. 2(a) to 2(c)). The result is a pack of particles with a random distribution and moderate compaction for which the porosity is 0.42 (figure. 2(d)). The DEM model is then imported into the LBM setup where flow is passing through the particles with constant velocities at boundaries (below and above the particle pack) to simulate a constant discharge of flow. Each simulation is continued until a steady state in flow characters (e.g. velocity distribution and fluid pressure) is obtained.

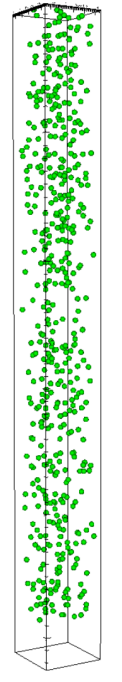

(a)

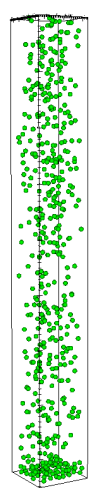

(b)

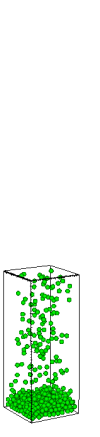

(c)

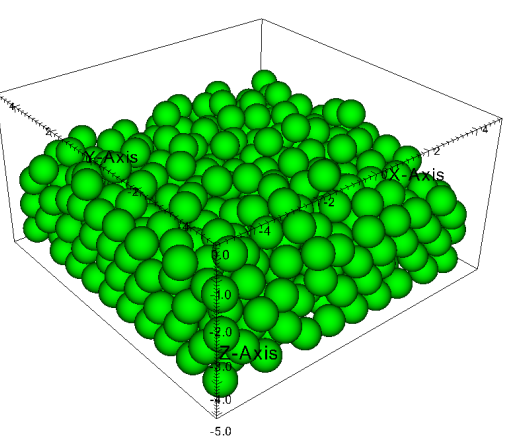

(d)
Figure 2: DEM simulation of particle deposition into a box $(a)$ to $c)$ ), generating a randomly distributed particulate medium $(d)$ )

\section{RESULTS AND DISCUSSION}

A wide range of flow velocities are examined to ensure that the results would cover the Darcy and nonDarcy flow conditions. To identify the various flow ranges, the fluid pressure loss versus Reynolds number is graphed (figure. 3(a)). The graph indicates the existence of a minimum pressure difference to initiate any flow. This conforms to what called the lower limit of applicability of Darcy law (Bear 1972). This means that there is a minimum (initial) gradient below which there is approximately no flow. The pressure drop threshold for flow initiation in the case of our study is about $2 \times 10^{-4} \mathrm{~Pa}$.

The linear relationship between pressure loss and fluid velocity suggests the range of Darcy condition. While the linear relationship is observed for a wide range of Reynolds numbers (from $R e \approx 10^{-2}$ to $\approx$ $1.5)$, the pressure loss curve is turning to a non-linear behaviour afterwards, until $R e \approx 12.6$, interpreted as the transition zone. This is in agreement with what was already suggested as the upper limit of Darcy range which is a Reynolds number between 1 to 10 (Bear 1972). The last part of the curve could be fitted with another equation as it has an obviously surged slope compared to the transition zone, suggesting the non-Darcy range. We however refrained to fit this part with a equation due to lack of numerous data points. The flow range identifications based on the analysis of the pressure drop curve are summarized in Table 1 . 
Table 1: Various conditions of the flow suggested by curve fitting over the fluid pressure difference graph (see figure. 3(a))

\begin{tabular}{lllll}
\hline Flow condition & Pre-Darcy & Darcy & Transition & Non-Darcy \\
Re range & $0-10^{-2}$ & $10^{-2}-1.5$ & $1.5-12.6$ & $12.6-18^{*}$ \\
Fitting & Constant & Linear & Non-Linear & unspecified \\
\hline
\end{tabular}

* The Re number of 18 is the largest Re number investigated in this study.

\subsection{Tortuosity}

The concept of tortuosity is relatively straightforward in definition, but quite controversial in practice. In the literature, the tortuosity is defined as either a geometrical parameter, or one associated to hydraulic, electrical or diffusive parameters (Ghanbarian et al. 2013). In a simple, but widely accepted descriptions, dimensionless parameter of hydraulic tortuosity is defined as:

$T=\frac{\langle\lambda\rangle}{L}$

where $\langle\lambda\rangle$ is the average length of the fluid paths and $L$ is the geometrical length of the sample.

In an analytically proven way, and shown to be valid later with several examinations (e.g. Jin et al. 2015), Duda et al. 2011 discussed the application of the following equation to calculate the tortuosity of flow:

$T=\frac{\sum v}{\sum v_{z}}$

where $v$ and $v_{z}$ denote the fluid velocity and the component of the fluid velocity in the direction of macroscopic flow, respectively.

The recent equation is however valid if both of following requirements are satisfied: the fluid must not be compressible, and the reentrant flow must not exist. The latter requirement is not met when countercurrents, i.e., flow streams heading in an opposite direction of macroscopic flow, are developed. As we are observing merge of countercurrents increasinly when the flow velocity decreases in the Darcy range, some modifications on the equation 12 are suggested. A preliminary modification could be to use the absolute magnitudes of $v_{z}$ in the denominator of the equation 12 , and another modification shall be to exclude all velocity vectors heading counterwards from both numerator and denominator of the tortuosity equation, resulting in the following modified equations, respectively:

$$
\begin{aligned}
T_{1} & =\frac{\sum v}{\sum\left|v_{z}\right|} \\
T_{2} & =\frac{\sum v_{v_{z}>0}}{\sum v_{z v_{z}>0}}
\end{aligned}
$$

with $v$ being the flow cell velocity magnitude, $v_{z}$ being the velocity vector component in the macroscopic flow direction (z-direction), $v_{v_{z}>0}$ being the flow velocity magnitude for the cells with positive velocity z-components, and $v_{z v_{z}>0}$ being the velocity zcomponents for those velocity vectors with positive $\mathrm{z}$-components.

Totuosity values as shown in figure. 3(b) indicate a substantial micro-scale behaviour of flow in porous media, which could be attributed to the origin of the Darcy law. Comparing the pre-Darcy, Darcy, and transitional ranges of flow acquired from pressurevelocity relationship (figure. 3(a)) with the tortuosity variations (figure. 3(b)), the flow conditions can be related to the stream pathlines. In fact, the most tortuos streams correspond to the pre-Darcy range, where no flow is initiated. It seems that the high tortuosity is the explanation why no flow is passing the medium, although a pressure gradient exists. That is due to pressure being dropped while flow is circulating throughout the medium, with no outcoming flow generated. With the pressure gradient exceeding the Darcy lower limit, streamlines begin to be shortened, causing smaller tortuosity values. The lowest tortuosity coincides with the end of Darcy range, where flow has reached the shortest possible pathline. From this point onward, as flow velocity increases, a higher rate of pressure gradient is required, as flow is no longer compensating the need for a more pressure drop with a shorter pathline. With the end of transitional range, where flow appears to enter the turbulent conditions, a slight rise in tortuosity is observed, apparently attributed to the turbulence of the flow. This behaviour corresponds with the last part of the pressure gradientvelocity curve, indicating the highest rate of pressure loss in the non-Darcy conditions.

\subsection{Drag Force}

A fluid moving relative to a solid apply a force on the solid boundary. This force is combined of two tangential and normal components relative to the solid surface. The tangenial component is caused by the viscosity and velocity gradient at the surface. The normal component is due to pressure variation along the surface. The vector, sum of these two components, integrated over the entire solid surface results in a force vector. The component of this force in the direction of relative velocity $(V)$ is called drag force, $D_{f}$.

Drag coefficient, $c_{d}$ is defined as:

$c_{d}=\frac{2 D_{f}}{\rho V^{2} A}$ 


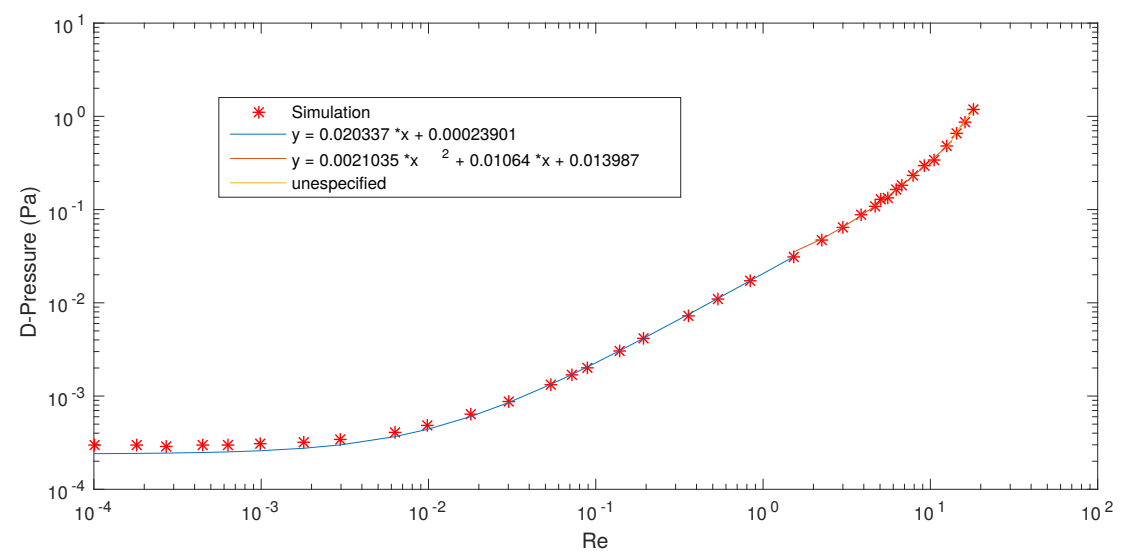

(a)

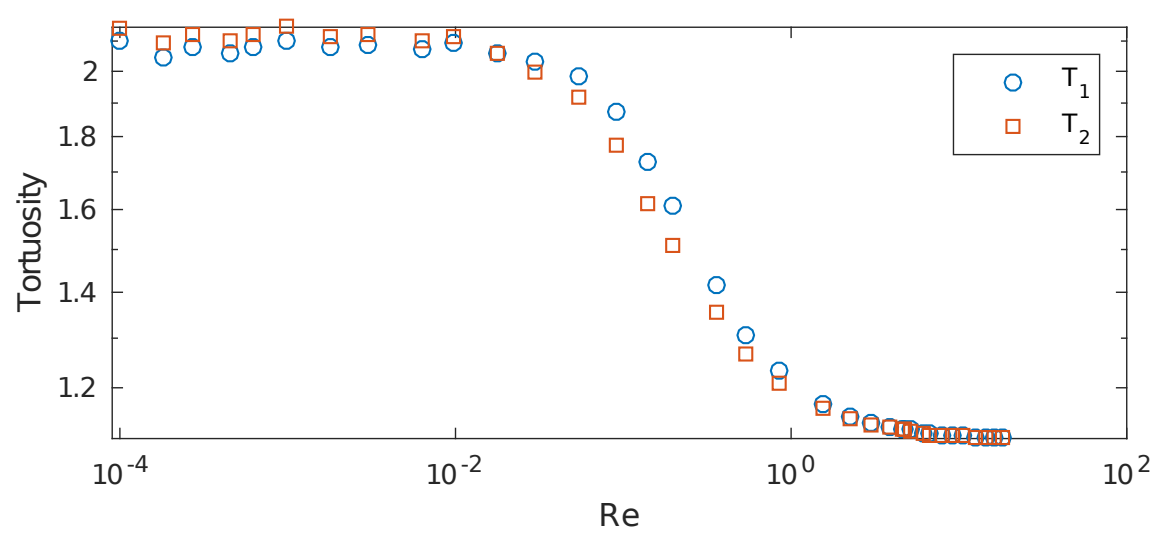

(b)

Figure 3: Pressure difference occurred in the flow passing through the particle pack showing the fitting equations for different parts of the graph (a) and the modified tortuosity of the flow (b), both as a function of Reynolds number

where $D_{f}$ and $V$ are as defined above, $\rho$ is the mass density of fluid, and $A$ is the frontal area normal to $V$. For spheres as applied in this study, $A$ could be simply taken as $\pi r^{2}$ (Bear 1972).

Figure 4 shows the variation of total drag forces applied on whole particle pack with the change of Reynolds number. The force values are slightly fluctuating around small values at pre-Darcy range (i.e., for $R e<10^{-2}$ ), apparently caused by the aforesaid circulating condition of flow. While the Darcy range provides a relatively constant increase in the drag force value, by flow's reaching to the transitional range $(R e \approx 1.5)$, drag forces surge with a high rate. The last part of the graph, belonging to the non-Darcy range, demonstrates a relatively sharper rising rate.

The drag coefficient, shown in figure 5, indicates a specific point at which the constant decreasing rate of $c_{d}$ converts to a increasing rate. This point appears to coincide with the end of Darcy range, i.e., $R e \approx 1.5$. Furthermore the increase of $c_{d}$ shows a relatively sharper rate along the non-Darcy range.

Drag coefficient is widely varied from large values of around 100 at lowest examined flow velocity to the smallest value of 0.08 occurring at the end of Darcy range. Its value, however, remains below 1.0 at whole range of Darcy to non-Darcy conditions.

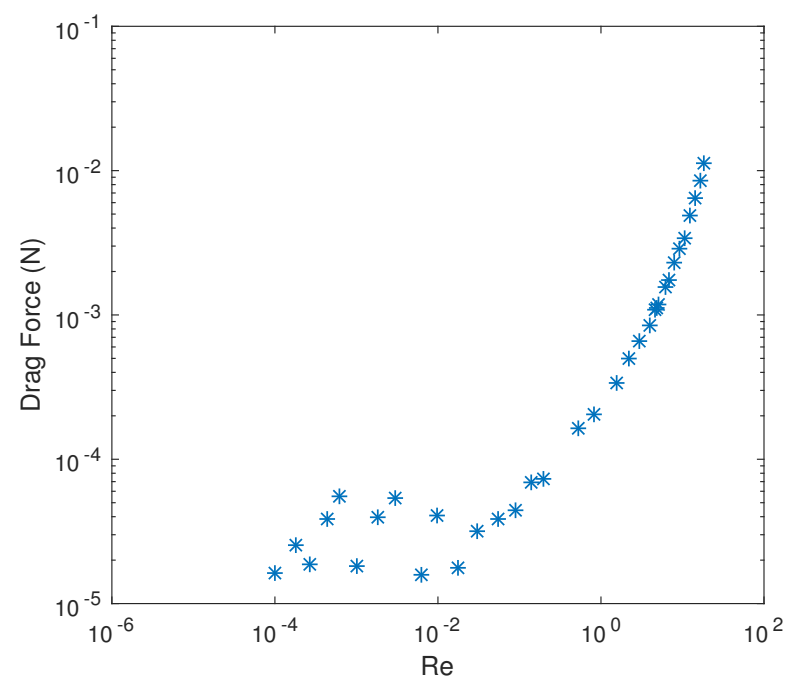

Figure 4: Drag force applied on the whole particle pack versus Reynolds number

\section{CONCLUSIONS}

The micro-scale investigation of fluid flow in porous media has been an interesting issue of research with 


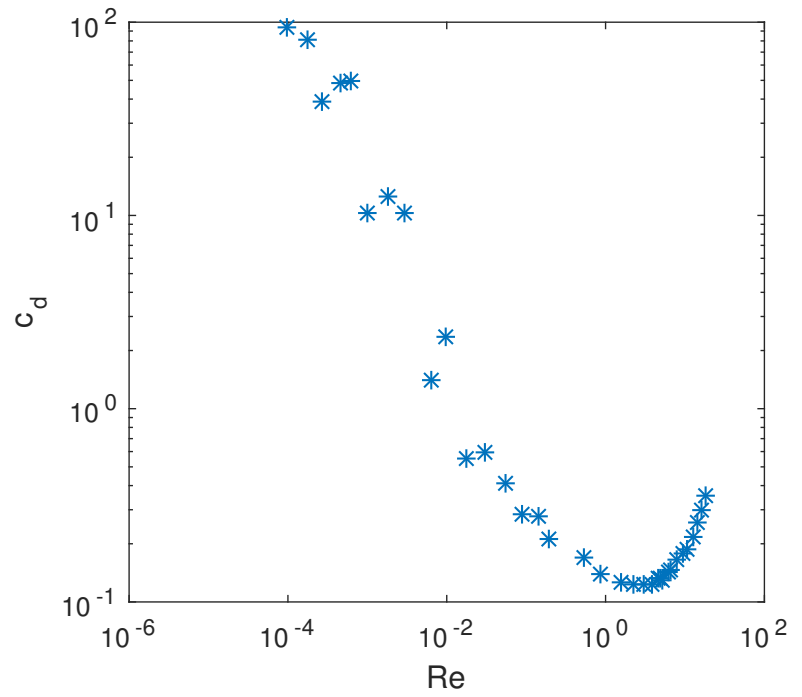

Figure 5: Drag coefficient versus Reynolds number

its significant applications in a large number of scientific and technological fields, such as geotechnical engineering, oil industry, pharmacological engineering, etc. The fluid behaviour in micro-scale is however, a complicated problem due to the complex porous medium structure, for either experimental or numerical investigations. Thanks to recent enhancements in computational powers, some aspects of fluid dynamics in porous media are revealed which have never been investigated in experiments. This study indicated a significant behaviour of fluids in pores which is the varying tortuosity, with the change of macroscopic flow velocity. It was shown that the flow has a varying tortuosity starting from the largest values at the beginning of Darcy range, gradually turning to the smallest values at the end of this range. This finding suggests a new lookout to the investigation of important concept of tortuosity, which has often been treated as a function of porosity, neglecting the flow velocity potential impacts. Furthermore, the variation of drag forces and the drag coefficient with the flow velocity was discussed, showing the coincident points of variations with the flow ranges.

The results could be improved with more discussion on the tortuosity calculations, especially when the countercurrent evolve in small velocities. Further investigation on the drag forces on the individual particles would also benefit when a erosion phenomenon is inspected. Although the method and the code has already been validated against experimental and analytical results (e.g. Galindo-Torres 2013), the findings would be stronger when validated using experimental examinations.

\section{ACKNOWLEDGEMENT}

This research received funding from the Discovery Project (DPI120102188), Hydraulic erosion of granular structures: experiments and computational simulations, funded by the Australian Research Council.

\section{REFERENCES}

Bear, J. (1972). Dynamics of fluids in porous media.

Buick, J. \& C. Greated (2000). Gravity in a lattice boltzmann model. Physical Review E 61(5), 5307.

Cundall, P. A. \& O. D. Strack (1979). A discrete numerical model for granular assemblies. Geotechnique 29(1), 47-65.

Duda, A., Z. Koza, \& M. Matyka (2011). Hydraulic tortuosity in arbitrary porous media flow. Physical Review E 84(3), 036319.

Freytag, I. \& W. L. Roque (2013). Influence of granular packing on porosity and tortuosity. Physical Review E 88(2), 023011.

Galindo-Torres, S. (2013). A coupled discrete element lattice boltzmann method for the simulation of fluid-solid interaction with particles of general shapes. Computer Methods in Applied Mechanics and Engineering 265, 107-119.

Galindo-Torres, S., D. Pedroso, D. Williams, \& L. Li (2012). Breaking processes in three-dimensional bonded granular materials with general shapes. Computer Physics Communications 183(2), 266-277.

Ghanbarian, B., A. G. Hunt, R. P. Ewing, \& M. Sahimi (2013). Tortuosity in porous media: a critical review. Soil science society of America journal 77(5), 1461-1477.

Guo, P. (2015). Lower and upper bounds for hydraulic tortuosity of porous materials. Transport in Porous Media 109(3), 659671.

He, X. \& L.-S. Luo (1997). Lattice boltzmann model for the incompressible navier-stokes equation. Journal of statistical Physics 88(3-4), 927-944.

Hecht, M. \& J. Harting (2010). Implementation of on-site velocity boundary conditions for d3q19 lattice boltzmann simulations. Journal of Statistical Mechanics: Theory and Experiment 2010(01), P01018.

Jin, Y., J. Dong, X. Li, \& Y. Wu (2015). Kinematical measurement of hydraulic tortuosity of fluid flow in porous media. International Journal of Modern Physics C 26(02), 1550017.

Martys, N. S. \& H. Chen (1996). Simulation of multicomponent fluids in complex three-dimensional geometries by the lattice boltzmann method. Physical review E 53(1), 743.

Matyka, M., A. Khalili, \& Z. Koza (2008). Tortuosity-porosity relation in porous media flow. Physical Review E 78(2), 026306.

Qian, Y., D. d'Humières, \& P. Lallemand (1992). Lattice bgk models for navier-stokes equation. EPL (Europhysics Letters) 17(6), 479.

Saomoto, H. \& J. Katagiri (2015). Particle shape effects on hydraulic and electric tortuosities: A novel empirical tortuosity model based on van genuchten-type function. Transport in Porous Media 107(3), 781-798.

Sun, Z., X. Tang, \& G. Cheng (2013). Numerical simulation for tortuosity of porous media. Microporous and Mesoporous Materials 173, 37-42.

Thorne, D. \& C. Michael (2006). Lattice boltzmann modeling. an introduction for geoscientists and engineers. $2 \mathrm{nd}$. $\mathrm{ed}$.

Wang, P. (2014). Lattice boltzmann simulation of permeability and tortuosity for flow through dense porous media. Mathematical Problems in Engineering 2014.

Wang, Y., S. Abe, S. Latham, \& P. Mora (2006). Implementation of particle-scale rotation in the 3-d lattice solid model. pure and applied geophysics 163(9), 1769-1785.

Xiao-Wu, T., S. Zu-Feng, \& C. Guan-Chu (2012). Simulation of the relationship between porosity and tortuosity in porous media with cubic particles. Chinese Physics B 21(10), 100201.

Zou, Q. \& X. He (1997). On pressure and velocity boundary conditions for the lattice boltzmann bgk model. Physics of Fluids (1994-present) 9(6), 1591-1598. 